\title{
Liver Resection After Selective Internal Radiation Therapy with Yttrium-90: Safety and Outcomes
}

\author{
Sebastian Mafeld ${ }^{1} \cdot$ Peter Littler $^{1} \cdot$ Hannah Hayhurst ${ }^{2} \cdot$ Derek Manas $^{2} \cdot$ Ralph Jackson $^{1} \cdot$ John Moir $^{2} \cdot$ Jeremy French $^{2}$
}

Published online: 26 March 2019

(C) The Author(s) 2019

\begin{abstract}
Introduction Selective internal radiotherapy (SIRT) with yttrium-90 (Y-90) is an intra-arterial therapy for hepatic malignancy in patients who are unsuitable for surgical resection. This treatment is considered palliative, although some patients can demonstrate a response that is adequate to facilitate surgical resection with curative intent.

Methods All patients who underwent liver resection post SIRT were reviewed. Data gathered included patient demographics, tumor type, surgical details, and post-operative outcomes.

Results Twelve patients underwent SIRT followed by liver resection ( 7 males and 5 females). Pathologies were hepatocellular carcinoma $(n=5)$, metastatic colorectal cancer $(n=5)$, and neuroendocrine tumor $(n=2)$. Lesional response (size, volume, and RECIST (response evaluation criteria in solid tumors)) was calculated and where appropriate functional liver remnant (FLR) is presented. Mean FLR increase was $264 \mathrm{~cm}^{3}$ (range - 123 to 909), and all cases demonstrated a partial response according to RECIST with a mean largest lesion volume reduction of $475 \mathrm{~cm}^{3}$ (range 14-1632). No post-SIRT complications were noted. Hepatectomy occurred at a mean of 322 days from SIRT treatment. Ninety-day morbidity was $67 \%(n=6)$, complications postsurgery were analyzed according to the Clavien-Dindo classification scale; a total of 15 events occurred in 6 patients. Ninety-day mortality of $11 \%(n=1)$.

Conclusion In selected cases, liver resection is possible post SIRT. As this can represent a potentially curative option, it is important to reconsider resection in the follow-up of patients undergoing SIRT. Post-operative complications are noted following major and extended liver resection. Therefore, further studies are needed to improve patient selection.
\end{abstract}

Keywords Selective internal radiotherapy - SIRT · Resection after SIRT · Y90 · Hepatic malignancy · Surgical resection · Radioembolization

\section{Introduction}

Selective internal radiotherapy (SIRT) with yttrium-90 (Y90) is an intra-arterial directed therapy for hepatic malignancy. Small $(20-60 \mu \mathrm{m})$ microspheres containing Y90, a beta emitter with a mean penetration range of $2.5 \mathrm{~mm}$ and a halflife of $64 \mathrm{~h}$ are infused into the target liver arteries in order to treat the tumors $[1,2]$. As hepatic tumors are mostly supplied by the arterial system, the delivery of Y90-coated microspheres into the liver arteries has a localized brachytherapy
John Moir

drjagmoir@doctors.org.uk

Jeremy French

Jeremy.French@nuth.nhs.uk

1 Department of Interventional Radiology, Freeman Hospital, Freeman Road, High Heaton, Newcastle upon Tyne NE7 7DN, UK

2 Department of Hepatobiliary Surgery, Freeman Hospital, Newcastle upon Tyne NE7 7DN, UK 
effect, while aiming to spare normal liver parenchyma which is supplied by the portal venous system. There are two commercially available forms of Y 90 microspheres; Theraspheres (BTG, London, UK) which produce glass-coated microspheres and SIR-Spheres (Sirtex, Sydney, Australia) which produce resin coated microspheres.

Most of the reported clinical evidence for SIRT is in the context of metastatic colorectal cancer (mCRC), hepatocellular carcinoma (HCC), and metastatic neuroendocrine tumors (mNET) [2]. The role of SIRT in the management of other hepatic malignancies is yet to be clearly defined [3]. For $\mathrm{mCRC}$, data demonstrates SIRT can improve progression-free survival in the liver, although results on its influence on overall survival and quality of life are evolving [4-7]. In HCC, SIRT may improve survival when compared with transarterial chemoembolization (TACE) and have potentially similar overall survival compared with sorafenib, but with an improved side effect profile [8,9]. For mNET, SIRT has been associated with a high response rate and improved survival [10].

Despite promising results, SIRT is considered a salvage therapy. A small cohort of patients can however have a response to SIRT which renders them potential candidates for curative surgical resection $[11,12]$. The experience with hepatic resection post SIRT is limited with less than 100 published cases. We retrospectively reviewed our experience with this technique.

\section{Materials and Methods}

Between 2011 and 2017, 138 Y90 deliveries were given in 107 patients. All patients underwent multi-disciplinary discussion prior to consideration of SIRT and were deemed surgically unresectable. Prior to administering SIRT, all patients received a planning angiogram and technetium ${ }^{99 \mathrm{~m}} \mathrm{Tc}$ macro aggregated albumin (T99mMAA) injection with subsequent gamma camera scintigraphy or CT-SPECT to detect shunting into the lungs or extrahepatic uptake. Follow-up imaging was planned at three monthly intervals post SIRT, but this did not occur in all patients. It is important to emphasize that similar to a previous study, SIRT was not intended a bridge to surgical resection at the time of treatment [12].

On subsequent follow-up imaging, a patient had response to SIRT whereby the surgical team thought surgical resection would be possible, then these cases were rediscussed at the multi-disciplinary tumor board. All patients who underwent hepatic resection post SIRT were reviewed.

Following resection, the 90-day post-operative mortality and morbidity were reviewed. In order to allow for comparison with a previous case series, morbidity was determined by Clavien-Dindo [13].

\section{Results}

\section{Patients}

A total of 12 (7 males, 5 females) patients underwent hepatic resection following SIRT, representing $11 \%$ of all SIRT cases. All patients had a normal serum bilirubin $(5.0$ to $17.0 \mathrm{mmol} / \mathrm{L}$ ) and had stopped chemotherapy at least 6 weeks prior to SIRT. Outcomes for 3 patients of these patients are not included in this review as one patient lacked follow up imaging data, another had a confounding procedure (portal vein embolization), and the third had a hemi hepatectomy with palliative intent due to symptomatic carcinoid syndrome with neuroendocrine metastases. Cases performed with curative intent were reviewed. A summary of case details is provided in Tables 1 , 2,3 and 4.

\section{SIRT}

Five patients underwent a single SIRT treatment while the remaining 4 patients had two treatments (this included two planned staged treatments and two repeat administrations). Eight patients had unilobar SIRT, and 1 patient received whole-liver SIRT. For the 2 patients who received a second SIRT administration, this occurred due to significant residual disease post initial SIRT, and following multi-disciplinary discussion, a second treatment was felt to be in the patients' best interests. No SIRT-related complication was seen. All lesions demonstrated a partial response to SIRT with a mean largest lesion volume reduction of $475 \mathrm{~cm}^{3}$ (range 14-1632). Where functional liver remnant (FLR) was felt to be a contributory reason to being unresectable, this was also calculated pre and post SIRT with a mean increase in FLR increase of $264 \mathrm{~cm}^{3}$ (range - 123 to 909). One patient has a decrease in FLR ($123 \mathrm{~cm} 3)$ as they received a bilobar treatment where the FLR was also exposed to SIRT. All patients who were initially unresectable on multi-disciplinary review had a reduction in lesion size and/or regression from critical vascular structures and/or increase in the necessary FLR. Patients proceeded to resection only after undergoing secondary multi-disciplinary review after SIRT response.

\section{Surgical Outcomes}

Mean time from SIRT to resection was 322 days (range 195703). Surgical procedures were extended right hemihepatectomy $(n=5)$, right hepatectomy $(n=1)$, extended left hepatectomy $(n=2)$, and right posterior sectionectomy $(n=1)$. Two cases required resection and reconstruction of the IVC. One case also underwent a metastasectomy at resection and another a roux-en-y hepaticojejunostomy. A R0 resection was achieved in 4 patients (44\%). 
Table 1 Demographics

\begin{tabular}{|c|c|c|c|c|c|c|c|c|}
\hline Patient & Gender & Diagnosis & $\begin{array}{l}\text { Tumor } \\
\text { volume } \\
\left(\mathrm{cm}^{3}\right)\end{array}$ & $\begin{array}{l}\text { Pre-SIRT } \\
\text { FLR }\end{array}$ & SIRT treatment & $\begin{array}{l}\text { Post SIRT } \\
\text { tumor } \\
\text { volume }\end{array}$ & $\begin{array}{l}\text { Post SIRT } \\
\text { FLR }\end{array}$ & $\begin{array}{l}\text { Change in } \\
\text { FLR }\end{array}$ \\
\hline 1 & $\mathrm{~F}$ & Metastatic colorectal & 35 & 625 & SIRTEX-right lobe & 21 & 1096 & +275 \\
\hline 2 & $\mathrm{~F}$ & $\begin{array}{l}\text { Metastatic } \\
\text { neuroendocrine }\end{array}$ & 109 & 240 & $\begin{array}{l}\text { SIRTEX-right lobe and } \\
\text { segment } 4 \text { (staged) }\end{array}$ & 41 & 420 & +180 \\
\hline 3 & M & $\mathrm{HCC}$ & 1150 & 656 & SIRTEX-right lobe & 136 & 819 & +163 \\
\hline 4 & $\mathrm{M}$ & $\mathrm{HCC}$ & 998 & 920 & SIRTEX-left lobe & 21 & 1126 & +206 \\
\hline 5 & $\mathrm{~F}$ & Metastatic colorectal & 33 & 490 & SIRTEX-right lobe & 9 & 936 & +446 \\
\hline 6 (Figure 1 and 2) & M & $\mathrm{HCC}$ & 1695 & 560 & $\begin{array}{l}\text { TheraSpheres-two } \\
\text { treatments } \\
\text { right lobe/segment } 4\end{array}$ & 63 & 1469 & +909 \\
\hline 7 & M & $\mathrm{HCC}$ & 508 & 1350 & TheraSpheres-right lobe & 306 & 1524 & +174 \\
\hline 8 & M & Metastatic colorectal & 22 & 347 & SIRTEX-right lobe & 5 & 493 & +146 \\
\hline 9 & $\mathrm{~F}$ & HCC (Fibrolamellar) & 350 & 1250 & $\begin{array}{l}\text { TheraSpheres-bilobar } \\
\text { split treatment }\end{array}$ & 20 & 1127 & $-123 *$ \\
\hline
\end{tabular}

Volumes calculated in $\mathrm{cm}^{3}$

*Due to bilobar treatment, some of the SIRT dose was likely given to the FLR resulting in a reduction in overall FLR

Table 2 Surgical details

Table 3 All Complications related to hepatic resection post SIRT

\begin{tabular}{|c|c|c|c|}
\hline Patient & $\begin{array}{l}\text { SIRT to } \\
\text { resection } \\
\text { (days) }\end{array}$ & Surgery & Margin \\
\hline 1 & 147 & Extended right hemihepatectomy & R0 \\
\hline 2 & 703 & $\begin{array}{l}\text { Right hemihepatectomy and metastectomy } \times 3 \text { and } \\
\text { right hemicolectomy }\end{array}$ & $\mathrm{R} 1$ \\
\hline 3 & 195 & Extended right hepatectomy & $\mathrm{R} 1$ \\
\hline 4 & 334 & Left hemi hepatectomy & $\mathrm{R} 0$ \\
\hline 5 & 130 & $\begin{array}{l}\text { Extended right hepatectomy, caudae lobectomy, } \\
\text { and IVC reconstruction }\end{array}$ & $\mathrm{R} 1$ \\
\hline 6 & 234 & $\begin{array}{l}\text { Extended right hemihepatectomy and roux-en-y } \\
\text { gastrojejunostomy (Figure 3) }\end{array}$ & $\mathrm{R} 1$ \\
\hline 7 & 678 & $\begin{array}{l}\text { Right posterior sectionectomy (segment 6/7) and } \\
\text { cholecystectomy }\end{array}$ & $\mathrm{R} 0$ \\
\hline 8 & 218 & Extended right hemihepatectomy & $\mathrm{R} 1$ \\
\hline 9 & 256 & $\begin{array}{l}\text { Extended left hepatectomy with resection and } \\
\text { reconstruction of IVC }\end{array}$ & $\mathrm{R} 0$ \\
\hline
\end{tabular}

\begin{tabular}{llc}
\hline Classification (Clavien-Dindo) & Event & Number \\
\hline I & & \\
II & Pneumonia & 2 \\
& Biliary Sepsis & 2 \\
& IVC Thrombus & 1 \\
IIIa & Bile Leak & 4 \\
& Biliary sepsis requiring percutaneous decompression & 1 \\
IIIb & Bilio-pleural fistula & 1 \\
& Hepatic vein stenosis (stented) & 1 \\
IVa & Respiratory failure & 1 \\
IVb & Multi organ failure & 1 \\
V & Death & 1 \\
\hline
\end{tabular}


Table 4 Surgical outcomes

\begin{tabular}{lllll}
\hline Patient & $\begin{array}{l}\text { Length } \\
\text { of stay }\end{array}$ & Complication & $\begin{array}{l}\text { 90-Day re- } \\
\text { admission }\end{array}$ & $\begin{array}{c}\text { Cause for re- } \\
\text { admission }\end{array}$ \\
\hline 1 & 79 (died) & $\begin{array}{l}\text { Hepatic vein stenosis (stented), biliary sepsis, } \\
\text { bile leak, pneumonia, } \\
\text { and multi-organ failure }\end{array}$ & NA & \\
2 & 20 & None & No & \\
3 & 6 & None & No & No \\
4 & 13 & None & No & Rigor with \\
5 & 30 & Bile leak/collection & Yes & biliary drain \\
6 & 79 & Bile leak, sepsis, respiratory failure, & and bilio-pleural fistula & No \\
7 & 10 & Hospital acquired pneumonia & No & \\
8 & 11 & Biliary sepsis & No & \\
9 & 67 & Bile leak, IVC thrombus & & \\
\hline
\end{tabular}

Median hospital stay was 16.5 days (range 6-79) with one re-admission (11\%). The 90-day morbidity rate was $67 \%$ ( $n=6)$ with 15 complications occurring in 6 patients, of which 10 complications were Clavien-Dindo grade 3 or above (Table 3$)$. Ninety-day mortality was $11 \% \quad(n=1)$ where a patient died 79 days post procedure following a complicated post-operative course with chest sepsis, bile leak, hepatic vein stenosis (stented), and subsequent multi-organ failure. No cases of post-hepatectomy liver failure were seen.

At mean follow-up at 878 days [range 79-2156], three patients died of which two developed radiographic progression, 5 patients remain alive and 1 was lost to follow up.

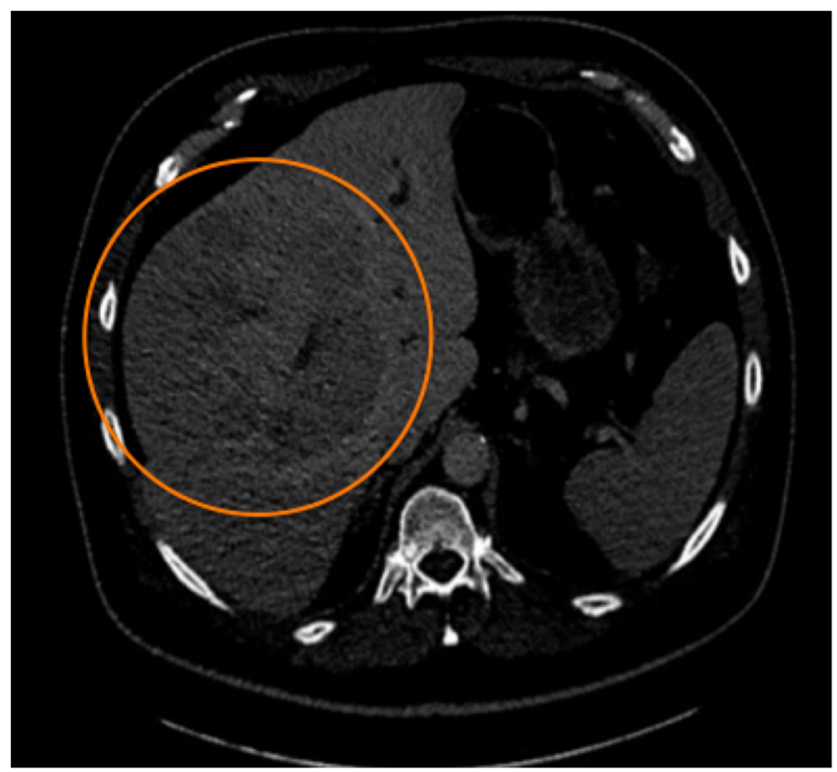

Fig. 1 Contrast-enhanced CT of a male patient with a 14-cm HCC (orange circle) with a tumor volume of $1695 \mathrm{~cm}^{3}$ and inadequate FLR $\left(560 \mathrm{~cm}^{3}\right)$ for surgical resection

\section{Discussion}

With hepatic malignancy drawing its blood supply primarily from the hepatic arterial system, the delivery of Y90 microspheres into this circulation is theorized to have preferential uptake in tumor cells rather than the normal liver tissue which is supplied by the portal venous system. As a beta emitter, the Y90 loaded microspheres have an effective radiotherapy range of about $2.5 \mathrm{~mm}$ and induce tumor cell injury through DNA damage [2]. Due to the short effective distance of the microspheres and portal venous blood supply, normal hepatic parenchyma should be relatively spared from the Y90 microsphere's radiotherapy effect [14].

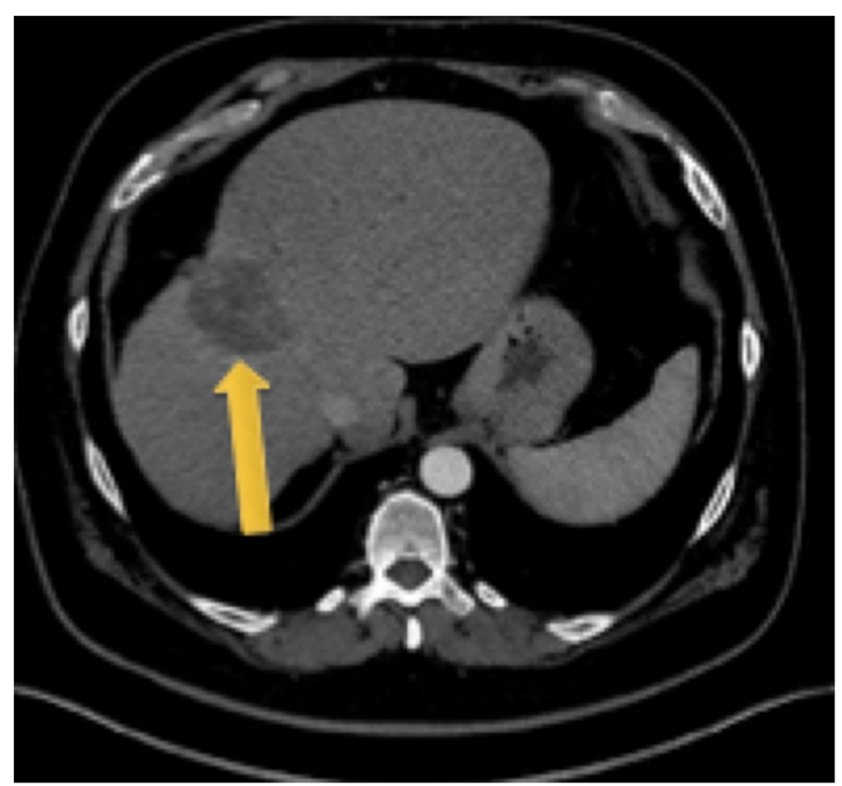

Fig. 2 Contrast-enhanced CT following two SIRT treatments. The tumor had decreased in size to a volume of $63 \mathrm{~cm}^{3}$, and the FLR increased to $1469 \mathrm{~cm}^{3}$ rendering the patient suitable for surgical resection 


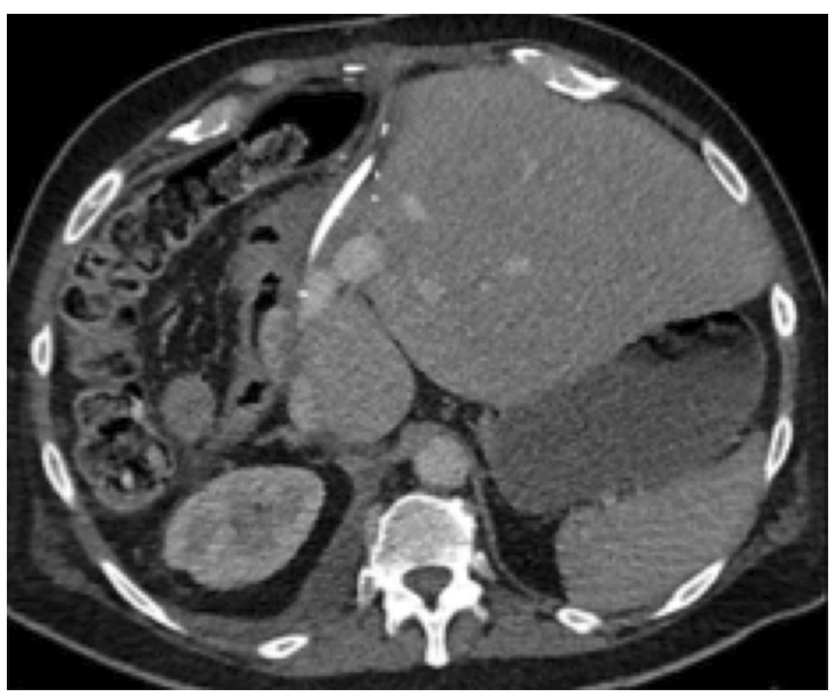

Fig. 3 Contrast-enhanced CT following extended right hepatectomy with roux-en-y hepaticojejunostomy

Surgical resectability is determined by the ability to safely achieve a surgical margin (R0) while preserving a residual adequate liver volume ((FLR) future liver remnant), typically 25 $30 \%$ of a healthy liver, but can increase up to $40 \%$ in patients with cirrhosis or after significant doses of chemotherapy [15]. If the FLR is the limiting factor for resection but is tumor free, portal vein embolization (PVE) can be considered to increase FLR. In the context of PVE failure, or bilobar disease, associating liver partition and portal vein ligation for staged hepatectomy (ALPPS) has been suggested as an alternative, albeit more controversial [16]. The disadvantage of both PVE and ALPPS is that while hepatic hypertrophy occurs, the initial disease process is left to progress and can even be stimulated by the hypertrophy process. Unilobar SIRT has the advantage of treating the liver tumors, which can result in volume reduction of the diseased lobe and contralateral lobe hypertrophy [17]. Furthermore, the hypertrophy occurs over an extended period of time, typically a number of months. This is reflected in the observed time from SIRT to surgery time (mean 322 days). It is thought this slower hypertrophy (compared to PVE or ALPPS) provides a better liver function per volume in the remnant liver. However, the degree of contralateral hypertrophy is typically less than can be achieved with PVE [18]. The precise mechanism for hypertrophy post SIRT is not known, but is likely multifactorial due to changes in portal venous hemodynamics and biochemical influences [19-24]. Except one The R0 resection rate of $44 \%$ in this series was despite the use of intraoperative ultrasound guiding clear resection of all of macroscopic disease. Of note, the positive margins identified after scrupulous histological examination consisted of limited microscopic infiltrative areas in areas not amenable to further surgical resection.

With its ability to both downstage disease and increase FLR, surgical resection after SIRT has in recent years been performed. Several case reports and small case series have suggested it can be a technically feasible option in carefully selected patients [25-30]. Only four larger series have also been published with a total experience of $n=64$ [11, 12, 31, 32]. In two of these series, resection was an afterthought following SIRT $[10,11]$ while Justinger et al. specifically selected marginally resectable cases and performed their surgical resections at a shorter interval (approximately 2 months) following SIRT [31]. Unless specifically intended at the time of SIRT, hepatic resection following SIRT is rare, with published literature indicating between 2 and $8 \%$ of patients receiving SIRT become surgically resectable $[10,11]$. More recent data from the SIRFLOX trial has suggested that up to $13 \%$ of patients with metastatic colorectal cancer can become resectable after SIRT [33]. Further analysis the SIRFLOX imaging data post SIRT has also suggested that more patients might become technically resectable than initially thought [34].

Direct comparison of our data with other series is challenging due to the heterogeneous nature of the available information. Several key themes however can be identified. Firstly, surgical resection after SIRT is associated with an increased morbidity compared to similar resections in patients who have not undergone SIRT, most commonly a bile leak. Our 90-day morbidity was in line with other studies ranging from 42 to $78 \%$ $[11,12]$. It is unclear whether this high morbidity is due to pretreatment with SIRT. Resections in cirrhotic patients with HCC can have morbidities of greater than 50\% [35]. However, in our cohort, the majority of hepatic complications occurred in the mCRC group. SIRT has been proposed to theoretically increase surgical morbidity as it can induce sinusoidal obstruction. This theory is purely based on the premise that sinusoidal obstruction can also be seen after oxaliplatin-based chemotherapy which has been shown to increase operative morbidity [7, 36, 37]. SIRT can also induce adhesion formation between the liver and adjacent structures which has in one case required partial diaphragmatic resection [7, 25]. One series with HCC patients alone reported a much lower complication rate (16\% ClavienDindo grade III or above) [32].

The impact of tumor type on surgical morbidity and the safest time for resection after SIRT are topics for further investigation. What this data and that of related studies do highlight is that SIRT should no longer be viewed only as a salvage therapy and patients' cases should re-reviewed at multi-disciplinary tumor boards for reconsideration of resection after SIRT. For patients with inadequate FLRs, PVE remains the standard of care. However, in circumstances where a tumor risks becoming unresectable due to the tumor growth that can be induced by PVE, SIRT may be a preferential treatment [38].

\section{Conclusion}

This study, while not changing suggested practice, contributes to the existing limited evidence indicating that hepatic resection 
after SIRT is technically possible albeit with a higher morbidity. It is unclear whether the higher morbidity is due to pretreatment with SIRT. Patients demonstrating a response to SIRT should be reconsidered for resection. Multi-disciplinary tumor board decision making remains key to patient selection. More research is needed to best identify candidates who may benefit from surgical resection following SIRT.

\section{Compliance with Ethical Standards}

Conflict of Interest All authors declare that they have no conflict of interest.

Ethical Approval All procedures performed in studies involving human participants were in accordance with the ethical standards of the institutional research committee and with the 1964 Helsinki declaration and its later amendments or comparable ethical standards.

Informed consent was obtained from all individual participants included in the study.

Open Access This article is distributed under the terms of the Creative Commons Attribution 4.0 International License (http:// creativecommons.org/licenses/by/4.0/), which permits unrestricted use, distribution, and reproduction in any medium, provided you give appropriate credit to the original author(s) and the source, provide a link to the Creative Commons license, and indicate if changes were made.

\section{References}

1. Bhangoo MS, Karnani DR, Hein PN, Giap H, Knowles H, Issa C, et al. Radioembolization with yttrium-90 microspheres for patients with unresectable hepatocellular carcinoma. J Gastrointest Oncol. 2015 Oct;6(5):469-78.

2. Kennedy A. Radioembolization of hepatic tumors. J Gastrointest Oncol. 2014;5(3):178-89.

3. Khajornjiraphan N, Thu NA, Chow PKH. Yttrium-90 microspheres: a review of its emerging clinical indications. Liver Cancer. 2015;4(1):6-15.

4. Wasan HS, Gibbs P, Sharma NK, Taieb J, Heinemann V, Ricke J, et al. First-line selective internal radiotherapy plus chemotherapy versus chemotherapy alone in patients with liver metastases from colorectal cancer (FOXFIRE, SIRFLOX, and FOXFIRE-Global): a combined analysis of three multicentre, randomised, phase 3 trials. Lancet Oncol. 2017;18(9):1159-71.

5. Townsend AR, Chong LC, Karapetis C, Price TJ. Selective internal radiation therapy for liver metastases from colorectal cancer. Cancer Treat Rev. 2016;50:148-54.

6. Gibbs P, Gebski V, Van Buskirk M, Thurston K, Cade DN, Van Hazel GA, et al. Selective internal radiation therapy (SIRT) with yttrium-90 resin microspheres plus standard systemic chemotherapy regimen of FOLFOX versus FOLFOX alone as first-line treatment of non-resectable liver metastases from colorectal cancer: the SIRFLOX study. BMC Cancer. 2014;1(14):897.

7. Garlipp B, Bruns CJ. The evidence for resection post-selective internal radiation therapy. Future Oncol. 2014;10(15s):49-52.

8. Zhang Y, Li Y, Ji H, Zhao X, Lu H. Transarterial Y90 radioembolization versus chemoembolization for patients with hepatocellular carcinoma: a meta-analysis. Biosci Trends. 2015;9(5):289-98.
9. Vilgrain V, Abdel-Rehim M, Sibert A, Ronot M, Lebtahi R, Castéra L, et al. Radioembolisation with yttrium-90 microspheres versus sorafenib for treatment of advanced hepatocellular carcinoma (SARAH): study protocol for a randomised controlled trial. Trials. 2014;15:474.

10. Devcic Z, Rosenberg J, Braat AJA, Techasith T, Banerjee A, Sze DY, et al. The efficacy of hepatic 90 Y resin radioembolization for metastatic neuroendocrine tumors: a meta-analysis. J Nucl Med Off Publ Soc Nucl Med. 2014;55(9):1404-10.

11. Henry LR, Hostetter RB, Ressler B, Bowser I, Yan M, Vaghefi H, et al. Liver resection for metastatic disease after y 90 radioembolization: a case series with long-term follow-up. Ann Surg Oncol. 2015;22(2):467-74.

12. Wright GP, Marsh JW, Varma MK, Doherty MG, Bartlett DL, Chung MH. Liver resection after selective internal radiation therapy with yttrium-90 is safe and feasible: a bi-institutional analysis. Ann Surg Oncol. 2017;24(4):906-13.

13. Dindo D, Demartines N, Clavien P-A. Classification of surgical complications. Ann Surg. 2004;240(2):205-13.

14. Kennedy AS, Nutting C, Coldwell D, Gaiser J, Drachenberg C. Pathologic response and microdosimetry of (90)Y microspheres in man: review of four explanted whole livers. Int J Radiat Oncol Biol Phys. 2004;60(5):1552-63.

15. Rotellar F, Pardo F, Martínez-Ortega P. The safety of resection postselective internal radiation therapy. Future Oncol. 2014;10(15s):53-5.

16. Cai Y-L, Song P-P, Tang W, Cheng N-S. An updated systematic review of the evolution of ALPPS and evaluation of its advantages and disadvantages in accordance with current evidence. Medicine (Baltimore) [Internet]. 2016 Jun 17 [cited 2017 Jun 3];95(24). Available from: http://www.ncbi.nlm.nih.gov/pmc/articles/PMC4998492/

17. Manas DM. Hypertrophy in the contralateral lobe post-selective internal radiation therapy. Future Oncol. 2014;10(15s):65-7.

18. Garlipp B, de Baere T, Damm R, Irmscher R, van Buskirk M, Stübs $\mathrm{P}$, et al. Left-liver hypertrophy after therapeutic right-liver radioembolization is substantial but less than after portal vein embolization. Hepatol Baltim Md. 2014;59(5):1864-73.

19. Teo J-Y, Allen JC, Ng DC, Choo S-P, Tai DWM, Chang JPE, et al. A systematic review of contralateral liver lobe hypertrophy after unilobar selective internal radiation therapy with Y90. HPB. 2015;n/a-n/a.

20. Jakobs TF, Saleem S, Atassi B, Reda E, Lewandowski RJ, Yaghmai $\mathrm{V}$, et al. Fibrosis, portal hypertension, and hepatic volume changes induced by intra-arterial radiotherapy with $90 y$ ttrium microspheres. Dig Dis Sci. 2008;53(9):2556-63.

21. Fernández-Ros N, Silva N, Bilbao JI, Iñarrairaegui M, Benito A, D'Avola D, et al. Partial liver volume radioembolization induces hypertrophy in the spared hemiliver and no major signs of portal hypertension. HPB. 2014;16(3):243-9.

22. Kawai M, Naruse K, Komatsu S, Kobayashi S, Nagino M, Nimura Y, et al. Mechanical stress-dependent secretion of interleukin 6 by endothelial cells after portal vein embolization: clinical and experimental studies. J Hepatol. 2002;37(2):240-6.

23. Hayashi H, Beppu T, Sugita H, Masuda T, Okabe H, Takamori H, et al. Serum HGF and TGF-betal levels after right portal vein embolization. Hepatol Res Off J Jpn Soc Hepatol. 2010;40(4):311-7.

24. Kusaka K, Imamura H, Tomiya T, Takayama T, Makuuchi M. Expression of transforming growth factor-alpha and -beta in hepatic lobes after hemihepatic portal vein embolization. Dig Dis Sci. 2006;51(8):1404-12.

25. Chua TC, Bester L, Akther J, Morris DL. Successful right hepatectomy after four treatments of yttrium-90 microspheres (SIRspheres) and concomitant FOLFOX as bridging therapy to resection of colorectal liver metastases. Anticancer Res. 2010;30(7):3005-7.

26. Neofytou K, Wasan H, Mudan S. Safety of redo hepatectomy for colorectal liver metastases after selective interarterial radiation therapy: a case report. Case Rep Surg [Internet] 2014 [cited 2017 Jun 1];2014. Available from: http://www.ncbi.nlm.nih.gov/pmc/ articles/PMC3971541/, 2014, 1, 5 
27. Sharma RA, Van Hazel GA, Morgan B, Berry DP, Blanshard K, Price D, et al. Radioembolization of liver metastases from colorectal cancer using yttrium-90 microspheres with concomitant systemic oxaliplatin, fluorouracil, and leucovorin chemotherapy. J Clin Oncol Off J Am Soc Clin Oncol. 2007;25(9):1099-106.

28. Sperling J, Justinger C, Schuld J, Ziemann C, Seidel R, Kollmar O. Intrahepatic cholangiocarcinoma in a transplant liver-selective internal radiation therapy followed by right hemihepatectomy: report of a case. World J Surg Oncol. 2014;12(1):198.

29. Wang LM, Jani AR, Hill EJ, Sharma RA. Anatomical basis and histopathological changes resulting from selective internal radiotherapy for liver metastases. J Clin Pathol. 2013;66(3):205-11.

30. Mafeld S, French J, Tiniakos D, Haugk B, Manas D, Littler P. Fibrolamellar hepatocellular carcinoma: treatment with yttrium-90 and subsequent surgical resection. Cardiovasc Intervent Radiol. 2018;41(5):816-20.

31. Justinger C, Kouladouros K, Gärtner D, Tatsch K, Reimer P, Rüdiger $\mathrm{T}$, et al. Liver resection after selective internal radiotherapy (SIRT): proof of concept, initial survival, and safety. J Surg Oncol. 2015;112(4):436-42.

32. Gabr A, Abouchaleh N, Ali R, Baker T, Caicedo J, Katariya N, et al. Outcomes of surgical resection after radioembolization for hepatocellular carcinoma. J Vasc Interv Radiol JVIR. 2018:27.

33. van Hazel GA, Heinemann V, Sharma NK, Findlay MPN, Ricke J, Peeters M, et al. SIRFLOX: randomized phase III trial comparing first-line mFOLFOX6 (plus or minus bevacizumab) versus mFOLFOX6 (plus or minus bevacizumab) plus selective internal radiation therapy in patients with metastatic colorectal cancer. J Clin Oncol. 2016;34(15):1723-31.

34. Garlipp B, Gibbs P, Van Hazel GA, Jeyarajah R, Martin RCG, Bruns CJ, et al. REsect: blinded assessment of amenability to potentially curative treatment of previously unresectable colorectal cancer liver metastases (CRC LM) after chemotherapy \pm RadioEmbolization (SIRT) in the randomized SIRFLOX trial. J Clin Oncol. 2017;35(15_suppl):3532-2.

35. Wei AC, Tung-Ping Poon R, Fan S-T, Wong J. Risk factors for perioperative morbidity and mortality after extended hepatectomy for hepatocellular carcinoma. Br J Surg. 2003;90(1):33-41.

36. Sangro B, Gil-Alzugaray B, Rodriguez J, Sola I, Martinez-Cuesta A, Viudez A, et al. Liver disease induced by radioembolization of liver tumors: description and possible risk factors. Cancer. 2008;112(7):1538-46.

37. Nordlinger B, Sorbye H, Glimelius B, Poston GJ, Schlag PM, Rougier P, et al. Perioperative chemotherapy with FOLFOX4 and surgery versus surgery alone for resectable liver metastases from colorectal cancer (EORTC intergroup trial 40983): a randomised controlled trial. Lancet Lond Engl. 2008;371(9617):1007-16.

38. Cucchetti A, Cappelli A, Ercolani G, Mosconi C, Cescon M, Golfieri R, et al. Selective internal radiation therapy (SIRT) as conversion therapy for unresectable primary liver malignancies. Liver Cancer. 2016;5(4):303-11.

Publisher's Note Springer Nature remains neutral with regard to jurisdictional claims in published maps and institutional affiliations. 